significantly associated with RAP ( $\mathrm{p}=0.55)$ or parasite infection, as concurrent infection was present in only 2 patients $(p=0.24)$.

Conclusion From this study, low grade inflammation, manifest by increased IELs, may be associated with RAP and also parasitic infection. $H$ pylori is not associated with parasite infection. However, as eosinophilia was not significantly associated with the condition further investigation is required to elucidate the potential involvement of innate immunity, including mast cells. Furthermore, there is no association between $H$ pylori infection and RAP. Funded by EU CONTENT Project (INCO-CT-2006-032136), CONICYT/ BM (RUE \#29) and Fondecyt \#1100654 (Chile).

Competing interests None declared.

\section{PTU-133 READY TO GO AND LET GO: PERSPECTIVES ON TRANSITION AND TRANSFER FROM PAEDIATRIC TO ADULT HEALTH CARE: A PAIRED PILOT SURVEY OF ADOLESCENT IBD PATIENTS AND THEIR PARENTS}

doi:10.1136/gutjnl-2012-302514c.133

N Rao, ${ }^{*}$ D Ashok, A Azaz, S Sebastian. Hull \& East Yorkshire NHS Trust, Hull, UK

Introduction National surveys report that transition care in inflammatory Bowel Disease is still not well developed. Although the general principles guiding transition of adolescents with chronic illnesses from paediatric to adult health care have been established, there are no studies to assess the transfer needs and concerns of adolescents with IBD and their carers. We aimed to gauge the perspectives of adolescents with IBD and their parents to determine their issues, concerns and expectations.

Methods A cross sectional survey of IBD patients starting transition process in a dedicated transition clinic and their parents was undertaken using a semi-structured questionnaire designed for self completion. Along with demographic and clinical information, respondents were asked to rate on a scale of $1-5$ (using Likert scale anchored by 1 -least important and 5 -very important and essential) their responses on their perceived importance on the aspects of preparation, self management skills, concerns regarding transfer and value of support services.

Results 20 patients (12 Crohn's and 8 Ulcerative Colitis) and their parents completed the survey independently. There was concordance in the responses of patients and their parents in rating highly the need for information, education and co-ordination of transfer process (Abstract PTU-133 table 1). The knowledge, empathy and accessibility were identified as key attributes for the transferring adult team (Abstract PTU-133 table 2). The highest rated concern for both adolescents and their parents was the perceived differences in performing tests such as endoscopy (mean score of $4.55 \pm 0.17$ and $4.65 \pm 0.23$ respectively). The adolescents favoured to attend appointments without parents before transfer (mean score

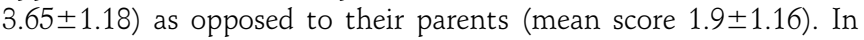
addition parents were concerned whether they will become less involved in care following transfer (mean score 4.15. The adolescents' favoured younger age of transition and transfer when compared to their parents. While dedicated adolescent services were described as beneficial by patients and their parents, only $50 \%$ of patients preferred to have joint appointments.

Conclusion This first pilot study demonstrates significant concerns about transition process needing addressing among adolescent IBD patients and their parents. Patients and parents differ in their rating of independence and self advocacy needed at the time of transfer. Tailoring transition to individual patient and parent needs without dedicated transition care teams may be challenging.

Competing interests None declared.
PTU-134 WHAT DO YOUNG PEOPLE AND PARENTS WANT FROM AN INFLAMMATORY BOWEL DISEASE (IBD) SERVICE?

doi:10.1136/gutjnl-2012-302514c.134

${ }^{1} \mathrm{R}$ Little, ${ }^{* 2} \mathrm{C}$ Imrie, ${ }^{3} \mathrm{~A}$ Derby, ${ }^{3} \mathrm{P}$ Gillespie, ${ }^{1} \mathrm{G}$ R Caddy, ${ }^{1} \mathrm{~T} \mathrm{C}$ Tham. ${ }^{1}$ Gastroenterology, Ulster Hospital, Dundonald, Belfast, Belfast, UK; ${ }^{2}$ Altnagelvin Hospital, Derry/Londonderry, UK; ${ }^{3}$ Northern Ireland branch, Crohns and Colitis UK, Belfast, UK

Introduction At present, there are guidelines from the US and Europe regarding the formation of transition clinics for adolescents with IBD. This includes a UK Inflammatory Bowel Disease (IBD) Standards guidance on optimal service provision for paediatric and adolescent care. However most of these guidelines come from intuitive reasoning and opinion, as there is a lack of data on what constitutes an ideal service for young patients with IBD. The aim of this study was to develop a comprehensive knowledge and understanding of the key service requirements of young people with IBD as well as their parents.

Methods Paediatric and adolescent patients age 6-18 years, were identified from databases in two teaching hospitals and from the membership of the N Ireland branch of Crohn's and Colitis UK, which is a patient support group. Anonymous questionnaires were sent to these patients and their parents separately. The questionnaires asked about their perceived quality of care, clinic care, general comments, input from specialists, support and information, plus any suggestions.

Results 105 questionnaires were sent and 51 responded (49\%); of these 21 were from patients and 30 from their parents. Over $84 \%$ were happy with the quality of care they are receiving. Reasons patients and parents were reluctant to attend clinics included: blood tests, nurse specialist or doctor not available, lack of car parking. $90 \%$ preferred to see the attending (Consultant) rather than a fellow. Nurse specialist, dietetics, specialist IBD surgeon, psychologist, skin/ eye specialist input was thought to be beneficial by $95 \%, 81 \%, 71 \%$, $59 \%$, and $45 \%$ respectively. The following support service and information were considered important: immediate contact with healthcare personnel for disease flare, support groups for young adults, insurance and financial advice, knowledge about IBD developments and research, email service, surgical input regarding stomas.

Conclusion The majority of young patients with IBD and their parents are satisfied with the care they are receiving. Support from specialist services such as nurse specialist, dietitians, specialist IBD surgeons, psychologist, plus rapid access to services when the disease flares were thought to be important by the patients and their parents. Knowledge of what these patients and their parents want will help to design an optimal IBD service.

Competing interests R Little: None Declared, C Imrie Conflict with: Mead Johnson, Falk Pharma, Nutricia, Warner Chilcott, SHS, Norgine, Wyeth, A Derby: None declared, P Gillespie: None declared, G Caddy: None declared, T Tham Speaker bureau with: Warner Chilcott, Shire, Conflict with: Abbott, MSD.

\section{PTU-135 INCIDENCE OF HP INFECTION IN CHILDREN AND THE ASSESSMENT OF HISTOLOGICAL STAINING PRACTICE}

doi:10.1136/gutjnl-2012-302514c.135

${ }^{1} \mathrm{Z}$ M Mead, ${ }^{1} \mathrm{~B}$ Vadgama, ${ }^{2} \mathrm{R}$ Mead, ${ }^{*}{ }^{1} \mathrm{D}$ Fowler, ${ }^{1} \mathrm{~S}$ Holden. ${ }^{1}$ Paediatric Histopathology, University Hospital Southampton NHS Trust, Southampton, UK; ${ }^{2}$ Gastroenterology, Hampshire Hospitals NHS Trust, Basingstoke, UK

Introduction The exact incidence of Helicobacter pylori (HP) in childhood is unknown. There is a causal link between HP and development of peptic ulcers, gastric adenocarcinoma and lymphoma. However there is little evidence and guidance on the clinical management of HP in children. The North American Society 